\title{
Cerebral Venous Sinus Thrombosis (CVST) in a Middle-Aged Female with Clustering of Risk Factors
}

\author{
Maria Prothasis ${ }^{1}$, Yash Gupte², Sourya Acharya ${ }^{3}$, Samarth Shukla ${ }^{4}$, Neema Acharya ${ }^{5}$ \\ ${ }^{1,2,3}$ Department of Medicine, Datta Meghe Institute of Medical Sciences, Wardha, Maharashtra, India. \\ ${ }^{4}$ Department of Pathology, Datta Meghe Institute of Medical Sciences, Wardha, Maharashtra, India. \\ ${ }^{5}$ Department of OBG, Datta Meghe Institute of Medical Sciences, Wardha, Maharashtra, India.
}

\section{INTRODUCTION}

Thrombosis of cerebral venous channel is a known complication of hypercoagulable states. Hyperhomocysteinaemia is a known hypercoagulable state. Obesity is a modern-day global epidemic. Disorders such as myocardial infarction (MI), stroke, and venous thromboembolism are on the rising trend and its increased morbidity and mortality is being associated with obesity. To date, however, the knowledge about the association between obesity and adult cerebral venous thrombosis (CVT) is sparse. We report a 44-year-old young morbidly obese metabolically unhealthy female who presented with headache, nausea, vomiting and giddiness. On evaluation, magnetic resonance venogram showed cerebral venous sinus thrombosis. On investigations, she had concomitant hyperhomocysteinaemia and metabolic syndrome.

Cerebral venous sinus thrombosis causing stroke in young adults is uncommon with various conditions precipitating it.1,2,3 Severe headache (70 - $90 \%$ ), focal lateralized signs ( $25 \%$ - $75 \%$ ), seizures (30 - $40 \%$ ) as well as behavioural symptoms such as delirium, amnesia, and disturbances in consciousness are the various associated clinical symptoms.

The known inherited hypercoagulable risk factors that cause CVST are gain of function mutations in the genes encoding factor $\mathrm{V}$ (factor $\mathrm{V}$ Leiden) and prothrombin, Protein C, S and antithrombin III deficiency. Hyperhomocysteinaemia, is a known risk factor for causing venous thrombosis of the lower limbs. However, till date there is no data available showing its role in causing cerebral venous thrombosis. The interaction between genetic and acquired determinants result in high plasma levels of total homocysteine (tHcy).4,5,6 Vitamins such as folic acid, pyridoxine, and cobalamin are involved in the metabolic pathways of homocysteine and its deficiencies represent the acquired determinants.

Venous thromboembolism (VTE) comprises of deep vein thrombosis of the leg and pulmonary embolism and obesity is now being recognised as one of the risk factors causing it. The risk of VTE is approximately increased to 2-fold in an individual with a body mass index (BMI) (calculated as weight in kilograms divided by height in meters squared) of 30 or more compared with a normal BMI $(<25)$, and higher BMIs increase more risk with approximately 3 times higher risk in individuals with a BMI greater than 40.7,8,9 Again obesity as a risk factor for CVST is less known.
Corresponding Author: Dr. Sourya Acharya, Professor and Head, Department of Medicine, Datta Meghe Institute of Medical Sciences, Sawangi Meghe, Wardha, Maharashtra, India.

E-mail: Souryaacharya74@gmail.com

DOI: $10.14260 / \mathrm{jemds} / 2021 / 605$

How to Cite This Article:

Prothasis M, Gupte Y, Acharya S, et al. Cerebral venous sinus thrombosis (CVST) in a middle aged female with clustering of risk factors. J Evolution Med Dent Sci 2021;10(34):2960-2963, DOI: 10.14260/jemds/2021/605

Submission 05-03-2021, Peer Review 24-06-2021, Acceptance 30-06-2021, Published 23-08-2021.

Copyright (C) 2021 Maria Prothasis et al. This is an open access article distributed under Creative Commons Attribution License [Attribution 4.0 International (CC BY 4.0)] 


\section{PRESENTATION OF CASE}

A 44-year-old young female, vegan with morbid obesity (weight $112 \mathrm{~kg}$, height $165 \mathrm{~cm}$, BMI 41.1, waist circumference $118 \mathrm{~cm}$ ) presented with complaints of headache, nausea, vomiting and giddiness since 3 days. The headache was continuous, diffuse with no aggravating of relieving factors. There was no history of fever, loss of consciousness, syncope, seizures, and weakness over limbs. There was no history of hypertension and diabetes mellitus too.

On examination, her pulse was $96 / \mathrm{min}$, BP - 130/80 mm $\mathrm{Hg}$. There were no signs of meningeal irritation, papilloedema or neurodeficit. Her other systemic examination was within normal limits. Her laboratory investigations revealed $\mathrm{Hb}$ of $8.9 \%$, mean corpuscular volume (MCV) - $119 \mathrm{fL}$, total leukocyte count (TLC) 7700/cumm. Peripheral smear showed macrocytosis. Fasting blood sugar was 108 mg \% (impaired fasting glucose), Lipid profile - serum cholesterol $287 \mathrm{mg} / \mathrm{dL}$, triglyceride $288 \mathrm{mg} / \mathrm{dL}$, low density lipoprotein (LDL) 198 $\mathrm{mg} / \mathrm{dL}$, high density lipoprotein (HDL) $40 \mathrm{mg} / \mathrm{dL}$. Her serum homocysteine levels were $46 \mu \mathrm{mol} / \mathrm{L}$ (normal 5 - $15 \mu \mathrm{mol} / \mathrm{L}$ ), serum Vitamin B12 levels 102 pg/ml (normal 200 - 900 $\mathrm{pg} / \mathrm{ml}$ ), thyroid profile was normal.
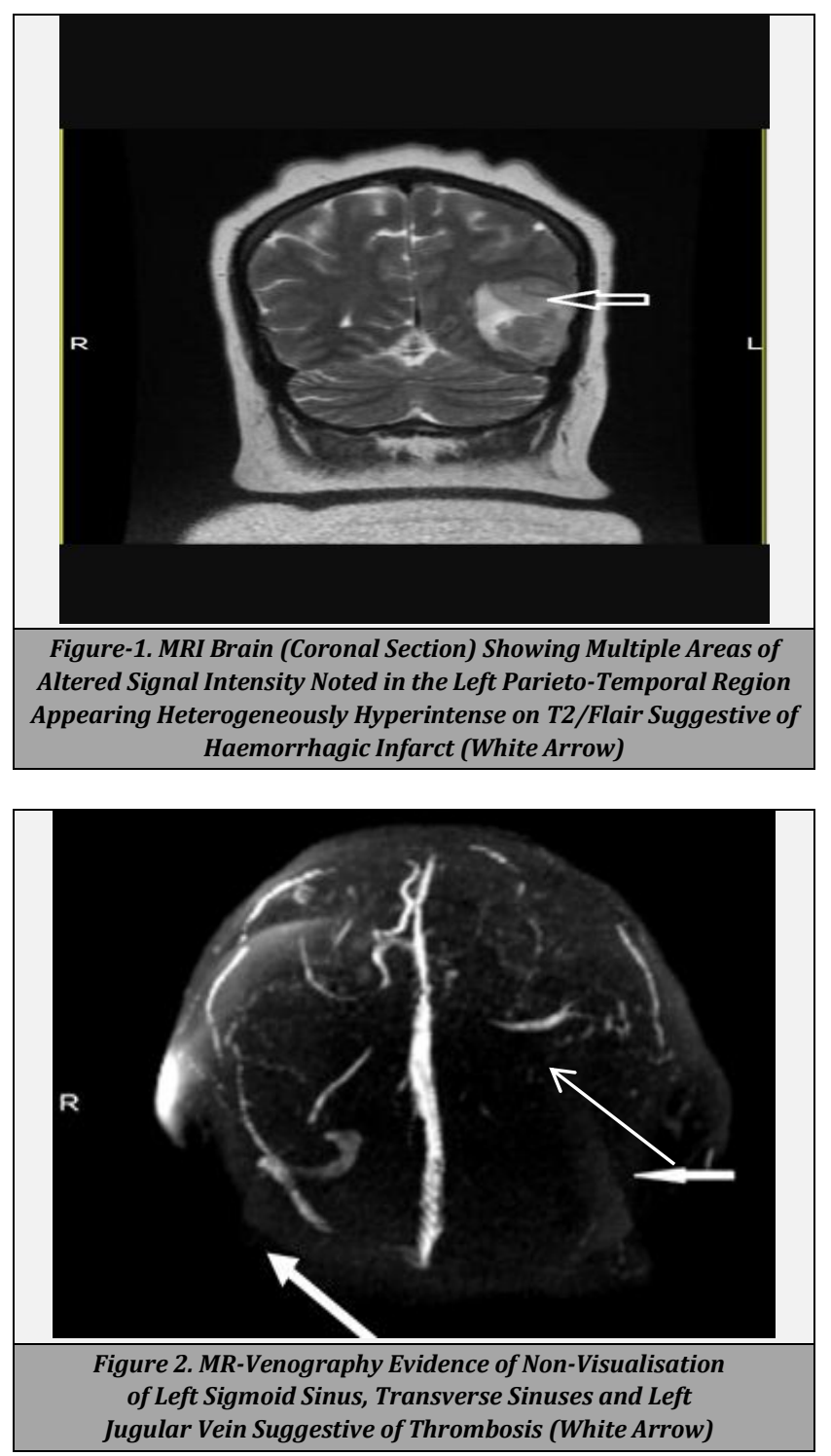

Magnetic resonance of imaging (MRI) of brain with magnetic resonance venography (MRV) revealed haemorrhagic infarct in left parietotemporal region and thrombosis of left sigmoid, transverse sinuses and the left jugular vein. (Figure 1, 2)

A diagnosis of CVST with metabolic unhealthy obesity and megaloblastic anaemia with hyperhomocysteinaemia was made. She was started on injection low molecular weight heparin (enoxaparin $0.6 \mathrm{ml}$ subcutaneously 12 hourly for 5 days which was overlapped with tablet warfarin $5 \mathrm{mg}$ titrated later to $7.5 \mathrm{mg}$ maintaining an international normalized ratio (INR) of 2.0. She was also started on inj. Vitamin B12 1000 micrograms IM once a day and tab folic acid $5 \mathrm{mg}$. She then improved clinically with effective regression of symptoms and was discharged with advice to continue inj. Vitamin B12 1000 micrograms IM every weekly for 2 months, then once a month life long, tablet folic acid $5 \mathrm{mg}$ once a day for 4 months, B6 10 mg per day and was asked to follow up after 7 days for INR evaluation.

\section{DISCUSSION}

Cerebral venous sinus thrombosis is a rare form of venous thromboembolism. ${ }^{10}$ Of all the types of stroke in young adults, CVST represents almost $0.5 \%-3 \% .{ }^{11,12}$ CVST has a female preponderance with a female to male ratio of $3: 1 .{ }^{13}$

Congenital thrombophilia is the most frequently associated factor. ${ }^{2}$ Homocystinaemia, factor $\mathrm{V}$ Leiden homozygous mutation, G20210A prothrombin gene and Methylene-Tetra-Hydro-Folate-Reductase 677TT mutations, protein $\mathrm{C}$ and $\mathrm{S}$ and anti-thrombin III deficiency, and positive anti-cardiolipin or anti-phospholipid antibodies are the various inherited prothrombotic risk factors for development of CVST. ${ }^{14,15}$

Methionine is an essential amino acid that generates homocysteine (Hcy) which is a sulphur containing amino acid. The Hcy concentration is maintained and regulated by transsulfuration or remethylation pathway. With the help of folate and vitamin B12 as a cofactor, Hcy is remethylated back to methionine with the enzyme methionine synthase. Methylenetetrahydrofolate reductase (MTHFR) and cystathione beta-synthase (CBS) enzyme activities can also affect Hcy levels. CBS controls the transsulfuration of Hcy to cystathione, and is dependent on Vitamin B6 as a cofactor. ${ }^{16}$ Increased levels of Hcy is a hypercoagulable state and a preclinical marker of stroke. Various prospective and retrospective clinical studies have provided evidence that Hcy may also cause stroke related thrombophilia. ${ }^{16,17}$

CVST is one of the commonest cause of stroke in India with pregnancy and puerperium being the most common aetiologies in females. However, it is uncommon in nonpuerperal setting. The pathological hallmark of CVST is haemorrhagic infarction. It is by and large a disease of the young and it can present in protean ways having a wide spectrum of clinical manifestations which include headache, altered sensorium, seizures, focal neurological deficits, papilloedema and cranial nerve palsies. Headache is most frequent and is often the earliest manifestation. ${ }^{18}$

The toxic effect of homocysteine on vascular endothelium can attenuate the oxidation of low-density lipoprotein cholesterol thus promoting thrombosis ${ }^{19}$ There still remains a 
putative association between CVST and hyperhomocysteinaemia. Hyperhomocysteinaemia has a strong association with vitamin B12 deficiency. The association between Hcy and B12 levels in a study revealed that, there was a significant difference between HCy and B12 at $95 \%$ confidence level. Thus, homocysteine value tends to be high if vitamin B12 value is low. On the contrary, however, when homocysteine is high, vitamin B12 need not be low. ${ }^{20}$ The study thus concluded that there is no evident association of vitamin B12 levels in patients of CVST and hyperhomocysteinaemia may not always be associated with low vitamin B12 level but it is definitely associated with CVST. Another study also had the similar conclusion. ${ }^{21}$ Our patient had lower Vitamin B12 and increased Hcy.

The change in coagulation factor levels is one mechanism which may increase the risk of thrombosis in obesity. Higher plasma concentrations of prothrombotic factors, such as plasminogen activator inhibitor 1 and Von Willebrand factor are found in obese women as compared to women having normal weight. ${ }^{22}$ Increased activated protein $\mathrm{C}$ resistance and higher concentrations of factor VIII, which are also the risk factors for thrombosis is associated with obesity. ${ }^{23}$ It is known that increased activated protein $C$ resistance is caused by use of oral contraceptives which may justify the synergistic effect of both risk factors that we observed. ${ }^{24}$

Of interest, idiopathic intracranial hypertension (IIH) also has obesity as a risk factor. They most often present with headache, decreased visual acuity and papilledema occurring due to impaired venous return in patients with chronic CVT. 25 It is found that similar to CVST, IIH is fairly more common in women compared to men, and the association between IIH and obesity is also more stronger in women.25,26 It has been hypothesized that IIH is caused by decreased outflow from the cerebral venous system, possibly owing to stenosis of the transverse sinuses or insufficiency of the valves in the jugular veins. ${ }^{25,26}$ Our patient did not have papilloedema ruling out $\mathrm{IHH}$, but she had jugular vein thrombosis.

Metabolic syndrome (MetS) is a distinct entity with conglomeration of vascular risk factors and metabolic abnormalities comprising of central obesity, atherogenic dyslipidaemia characterized mainly by elevated triglycerides and decreased high-density lipoproteins, high blood pressure, and hyperglycemia.27 MetS has been associated with an increased risk of prevalent stroke. In the National Health and Nutrition Examination Survey among 10,357 subjects, the prevalence of MetS was significantly higher in persons with a self-reported history of stroke (43.5\%) than in subjects with no history of stroke (22.8\%). MetS was independently associated with stroke history in all ethnic groups and in both sexes (OR, 2.16; $95 \%$ CI, 1.48 to 3.16). ${ }^{28}$ Clinical studies have confirmed that frequency of MetS is markedly higher in patients with a history of atherothrombotic or non-embolic ischemic stroke. ${ }^{28-30}$ Long-term follow-up population-based studies have proven that healthy individuals having MetS are at a significantly increased risk for having major cardiovascular events, including stroke. Our case also had MetS.

\section{CONCLUSIONS}

Cerebral venous sinus thrombosis in our case had multiple coexisting risk factors like obesity, metabolic syndrome, hyperhomocysteinaemia and B12 deficiency. This case was rare to have clustering of more than one risk factors. Any obese female who presents with symptoms of CVST should be investigated for possible occurrence of other risks.

Financial or other competing interests: None.

Disclosure forms provided by the authors are available with the full text of this article at jemds.com.

\section{REFERENCES}

[1] Ageno W, Becattini C, Brighton T, et al. Cardiovascular risk factors and venous thromboembolism: a meta-analysis. Circulation 2008;117(1):93-102.

[2] Stam J. Thrombosis of the cerebral veins and sinuses. New Engl J Med 2005;352(17):1791-8.

[3] Allroggen H, Abbott RJ. Cerebral venous sinus thrombosis. Postgrad Med J 2000;76(891):12-15

[4] Brækkan SK, Hald EM, Mathiesen EB, et al. Competing risk of atherosclerotic risk factors for arterial and venous thrombosis in a general population: the Tromso study. Arterioscler Thromb Vasc Biol 2012;32(2):487-91.

[5] Tsai AW, Cushman M, Rosamond WD, et al. Cardiovascular risk factors and venous thromboembolism incidence: the longitudinal investigation of thromboembolism etiology. Arch Intern Med 2002;162(10):1182-9.

[6] Holst AG, Jensen G, Prescott E. Risk factors for venous thromboembolism: results from the Copenhagen City Heart Study. Circulation 2010;121(17):1896-903.

[7] Pomp ER, le Cessie S, Rosendaal FR, et al. Risk of venous thrombosis: obesity and its joint effect with oral contraceptive use and prothrombotic mutations. $\mathrm{Br} \mathrm{J}$ Haematol 2007;139(2):289-96.

[8] Zuurbier SM, Arnold M, Middeldorp S, et al. Risk of cerebral venous thrombosis in obese women. JAMA Neurol 2016;73(5):579-84.

[9] Seheult JN, Chibisov I. A case of unexplained cerebral sinus thrombosis in a 22 year-old obese Caucasian woman, Laboratory Medicine 2016;47(3):233-40.

[10] Bousser MG, Crassard I. Cerebral venous thrombosis, pregnancy and oral contraceptives. Thromb Res 2012;130(Suppl 1):S19-S22.

[11] Bousser MG, Ferro JM. Cerebral venous thrombosis: an update. Lancet Neurol 2007;6(2):162-70.

[12] Ruiz-Sandoval JL, Chiquete E, Bañuelos-Becerra LJ, et al. Cerebral venous thrombosis in a Mexican multicenter registry of acute cerebrovascular disease: The RENAMEVASC study. J Stroke Cerebrovasc Dis 2012;21(5):395-400.

[13] Ferro JM, Canhão P, Stam J, et al. Prognosis of cerebral vein and dural sinus thrombosis: results of the International Study on Cerebral Vein and Dural Sinus Thrombosis (ISCVT). Stroke 2004;35(3):664-70.

[14] Roach ES, Golomb MR, Adams R, et al. Management of stroke in infants and children: a scientific statement from a Special Writing Group of the American Heart 
Association Stroke Council and the Council on Cardiovascular Disease in the Young. Stroke 2008;39(9):2644-91.

[15] Wysokinska EM, Wysokinski WE, Brown RD, et al. Thrombophilia differences in cerebral venous sinus and lower extremity deep venous thrombosis. Neurology 2008;70(8):627-33.

[16] Maxwell SR. Coronary artery disease--free radical damage, antioxidant protection and the role of homocysteine. Basic Res Cardiol 2000;95(Suppl 1):I6571.

[17] Graham IM, O'Callaghan P. Vitamins, homocysteine and cardiovascular risk. Cardiovasc Drugs Ther 2002;16(5):383-9.

[18] Bousser MG, Chiras J, Bones J, et al. Cerebral venous thrombosis. A review of 38 cases. Stroke 1985;16(2):199213.

[19] Clarke R, Daly L, Robinson K, et al. Hyperhomocysteinemia - an independent risk factor for vascular disease. New Eng J Med 1991;324(17):1149-55.

[20] Misra UK, Kalita J, Bansal V. D- dimer is useful in the diagnosis of cortical venous thrombosis. Neurology India 2009;57(1):50-4.

[21] Harale M, Alurkar A, Basavaraj A, et al. Co-relation of cerebral venous sinus thrombosis with Vitamin B12 and homocysteine levels in a tertiary care centre. J Association of Physicians of India 2019;67(1):34-7.

[22] De Pergola G, De Mitrio V, Giorgino F, et al. Increase in both pro-thrombotic and anti-thrombotic factors in obese premenopausal women: relationship with body fat distribution. Int $\mathrm{J}$ Obes Relat Metab Disord 1997;21(7):527-35.

[23] Christiansen SC, Lijfering WM, Naess IA, et al. The relation-ship between body mass index, activated protein $\mathrm{C}$ resistance and risk of venous thrombosis. J Thromb Haemost 2012;10(9):1761-7.

[24] Rosing J, Middeldorp S, Curvers J, et al. Low-dose oral contraceptives and acquired resistance to activated protein C: a randomised cross-over study. Lancet 1999;354(9195):2036-40.

[25] Nedelmann M, Kaps M, Mueller-Forell W. Venous obstruction and jugular valve insufficiency in idiopathic intracranial hypertension. J Neurol 2009;256(6):964-9.

[26] Wakerley BR, Tan MH, Ting EY. Idiopathic intracranial hypertension. Cephalalgia 2015;35(3):248-61.

[27] Eckel RH, Grundy SM, Zimmet PZ. The metabolic syndrome. Lancet 2005;365(9468):1415-28.

[28] Ninomiya JK, L'Italien G, Criqui MH, et al. Association of the metabolic syndrome with history of myocardial infarction and stroke in the Third National Health and Nutrition Examination Survey. Circulation 2004;109(1):42-6.

[29] Milionis HJ, Rizos E, Goudevenos J, et al. Components of the metabolic syndrome and risk for first-ever acute ischemic non-embolic stroke in elderly subjects. Stroke 2005;36(7)p:1372-6.

[30] Seung-Han S, Sacco RL, Boden-Albala B, et al. Abdominal obesity and risk of ischemic stroke. The Northern Manhattan Stroke Study. Stroke 2003;34(7):1586-92. 